\title{
On well-posedness for some thermo-piezo-electric coupling models
}

\author{
A. J. Mulholland ${ }^{a *}$, R. Picard ${ }^{b}$, S. Trostorff ${ }^{b}$ and M. Waurick ${ }^{b}$
}

There is an increasing reliance on mathematical modelling to assist in the design of piezoelectric ultrasonic transducers since this provides a cost-effective and quick way to arrive at a first prototype. Given a desired operating envelope for the sensor the inverse problem of obtaining the associated design parameters within the model can be considered. It is therefore of practical interest to examine the well-posedness of such models. There is a need to extend the use of such sensors into high temperature environments and so this paper shows, for a broad class of models, the well-posedness of the magneto-electro-thermo-elastic problem. Due to its widespread use in the literature, we also show the well-posedness of the quasi-electrostatic case. Copyright (c) 0000 John Wiley \& Sons, Ltd.

Keywords: piezoelectric; well-posedness; Maxwell equations; thermal

\section{Introduction.}

Piezoelectric structures can receive electrical energy and use this to alter their mechanical and thermal properties (and vice versa) and hence they are ideally placed for use in smart materials that can be automatically adjusted to assist in the vibration and thermal stress control of structures [32], as actuators [38], or as energy harvesters [42]. To assist in the design of such technology there is an increasing reliance on mathematical models as a cost-effective and fast way to arrive at a first prototype [43]. The primary use of these piezoelectric materials is in ultrasonic transducers. In transmission mode an electrical signal is sent in to the device and the resulting mechanical vibration causes an ultrasound wave to be transmitted through the material of interest. Having traversed this material this mechanical wave is then received by the transducer and converted back to an electrical signal for processing. Typical applications of this technology can be found in medical imaging and non-destructive testing of safety critical structures [35, 36, 37]. The well-posedness of these models is therefore of practical interest if these models are to be used in considering inverse problems centred on optimising the material parameters and other design parameters to meet some pre-specified operational quality of the device [2, 25, 26, 27, 29, 28, 41, 40, 39]. The well-posedness of the forward problem in piezoelectric material modelling is also essential if one wants to consider the inverse problem of obtaining the piezoelectric material tensors from experimental measurements [15, 19]. A modelling assumption that is often employed in order to reduce the size of the model is the so-called quasi-electrostatic approach [10, 11, 13, 20]. Since the electrical waves travel at a far faster speed than the mechanical waves within a piezoelectric material then this separation in time scales is used to focus attention on the timescale of the mechanical waves by assuming that the electrical

a Department of Mathematics and Statistics, University of Strathclyde, Glasgow, U.K.

b Department of Mathematics, Technische Universitaet Dresden, Dresden, Germany

* Correspondence to: Department of Mathematics and Statistich Unipirsity\&fStrathclyde, Livingstone Tower, 26 Richmond Street, Glasgow G1 1XH, U.K., Tel: ++44(0)141548 2971, Fax: ++44(0)1415483345, email: anthony.mulholland@strath.ac.uk 
Mathematical

Methods in the

Applied Sciences

A. N. Mulholland et al.

activity is instantaneous (so the electrical potential is spatially uniform). As well as simplifying the analysis this approach can also help to reduce the computational time in numerical simulations of electromechanical waves propagating in a piezoelectric material [14]. Given the widespread use of this approximation the question of the well-posedness of the quasi-electrostatic model of a piezoelectric material has been studied $[16,17,18]$. It is also possible to incorporate dissipative loss terms in the formulation and consider the existence and regularity of the model solution [22] and thermal effects [24]. A useful summary of the literature that has examined this well-posedness for a range of boundary conditions is provided by Akamatsu and Nakamura [1]. They prove that in a bounded domain with a Lipschitz boundary the initial-boundary value problem is wellposed. The initial conditions pertain to the mechanical displacement and its time derivative, and boundary conditions are stated in terms of the mechanical displacement, the mechanical stress, the electric potential and the electric displacement; this is a typical scenario for a piezoelectric transducer operating in reception mode. When a piezoelectric device is being tested it is normally immersed in a water bath and its transmission performance assessed by using a receiving hydrophone at some distance from the piezoelectric device. The existence and uniqueness of solutions to this fluid-solid interaction problem have very recently been settled using a potential method and the theory of pseudodifferential equations [9]. The situation where the piezoelectric material is in contact with a surface and undergoing anti-plane motion has also been investigated [4, 23]. In this situation the uniqueness and existence of a weak solution is proved. The continuous dependence of the solution on the data has also been shown [5] and this has been used to develop a numerical solution of the problem. This piezoelectric problem was recently extended to consider the existence and uniqueness when coupled with the thermal effects [6]. Such models are needed to assist in the design of smart ceramic materials that can adjust their mechanical properties as the temperature of their environment fluctuates; in the non-destructive testing application, for example, there is an increasing demand for ultrasonic transducers that can withstand high temperatures. The above work is based on the quasi-electrostatic approximation and the well-posedness of the non-stationary piezoelectric system, wherein the Maxwell equations are involved, has also been considered [3]; one simplification that is often used however is to study the time harmonic case [21]. The emergence of smart materials has very recently led researchers to extend the model to include magnetic and thermal effects and the well-posedness of the magneto-electro-thermo-elastic problem has been very recently considered [7, 30, 34]. By non-dimensionalising the problem and identifying a small parameter this dynamic model was systematically reduced to the quasi-electrostatic case. Note that they assumed that all the coefficients were bounded and satisfied some conditions warranting positive definiteness. This then permitted the use of semi-group theory to settle the well-posedness issue. The resulting electrical and magnetic potentials enabled consideration of the energy transfer in the material and this therefore has applications in the design of energy harvesting materials. In this paper we will consider a similar model but our methodology will apply to a broader class of problems (for example the operators here could be non-local of convolution type) and will not be restricted to the multiplication operators as in this previous work. The coefficients could be fully anisotropic, inhomogeneous and even could - for example - be non-local. In some cases material behaviour may be better described by an averaging influence of the neighborhood. To be concrete, operator coefficients could show a behaviour like

$$
u \mapsto K u
$$

with

$$
(K u)(x):=\int_{w} k(x-y) u(x) d x
$$

where $k$ is a kernel function, e.g. generated by the Gaussian distribution, and $x+[w], w \subseteq \mathbb{R}^{3}$, a window of influence impacting on the behaviour at point $x$. The considerations are limited in so far as only linear material behaviour is considered. Thus, for example, the non-linear effects of the temperature, which are known to limit the piezo-electric effect, see the plate case e.g. [8], [33] and the references cited therein, are clearly beyond the scope of this ground-laying study of the general linear case.

\section{The System Equations of Thermo-Piezo-Electro-Magnetism.}

Let $\Omega \subseteq \mathbb{R}^{3}$. The system of Thermo-Piezo-Electro-Magnetism is a coupled system consisting of the equation of elasticity, Maxwell's equations and the equation of heat conduction. Whiley \&ouns sout, we denote by $\partial_{0}$ the derivative with respect to time. 
The equation of elasticity is given by

$$
\partial_{0}^{2} \varrho_{*} u-\operatorname{Div} T=F_{0},
$$

where $u: \mathbb{R} \times \Omega \rightarrow \mathbb{R}^{3}$ describes the displacement of the elastic body $\Omega, T: \mathbb{R} \times \Omega \rightarrow \operatorname{sym}\left[\mathbb{R}^{3 \times 3}\right]$ denotes the stress tensor, which is assumed to attain values in the space of symmetric matrices. The function $\varrho_{*}: \Omega \rightarrow \mathbb{R}$ stands for the density of $\Omega$ and $F_{0}: \mathbb{R} \times \Omega \rightarrow \mathbb{R}^{3}$ is an external force term. Maxwell's equation are given by

$$
\begin{aligned}
& \partial_{0} B+\operatorname{curl} E=F_{3}, \\
& \partial_{0} D-\operatorname{curl} H=F_{2}-\sigma E .
\end{aligned}
$$

Here, $B, D, E, H: \mathbb{R} \times \Omega \rightarrow \mathbb{R}^{3}$ denote the magnetic flux density, the electric displacement, the electric field and the magnetic field, respectively. The functions $F_{2}, F_{3}: \mathbb{R} \times \Omega \rightarrow \mathbb{R}^{3}$ are given source terms and $\sigma: \Omega \rightarrow \mathbb{R}$ denotes the resistance. Finally, the equation of heat conduction is given by

$$
\partial_{0} \Theta_{0} \eta+\operatorname{div} q=F_{4}
$$

where $\eta: \mathbb{R} \times \Omega \rightarrow \mathbb{R}$ denotes the entropy density, $q: \mathbb{R} \times \Omega \rightarrow \mathbb{R}^{3}$ is the heat flux, $F_{4}: \mathbb{R} \times \Omega \rightarrow \mathbb{R}$ is an external heat source and $\Theta_{0}: \Omega \rightarrow \mathbb{R}$ is the reference temperature. Of course, all these equations need to be completed by suitable material laws, where also the coupling will occur. As it will turn out, the system can be written in the following abstract form

$$
\left(\partial_{0} M_{0}+M_{1}+\left(\begin{array}{cccccc}
0 & - \text { Div } & 0 & 0 & 0 & 0 \\
- \text { Grad } & 0 & 0 & 0 & 0 & 0 \\
0 & 0 & 0 & -\operatorname{curl} & 0 & 0 \\
0 & 0 & \text { curl } & 0 & 0 & 0 \\
0 & 0 & 0 & 0 & 0 & \operatorname{div} \\
0 & 0 & 0 & 0 & \operatorname{grad} & 0
\end{array}\right)\right)\left(\begin{array}{c}
v \\
T \\
E \\
H \\
\Theta_{0}^{-1} \theta \\
q
\end{array}\right)=F
$$

for suitable bounded operators $M_{0}, M_{1}$ on the Hilbert space $H:=L^{2}(\Omega)^{3} \oplus \operatorname{sym}\left[L^{2}(\Omega)^{3 \times 3}\right] \oplus L^{2}(\Omega)^{3} \oplus L^{2}(\Omega)^{3} \oplus L^{2}(\Omega) \oplus$ $L^{2}(\Omega)^{3}$. Here, $v:=\partial_{0} u$ and $\theta: \mathbb{R} \times \Omega \rightarrow \mathbb{R}$ denotes the temperature. Of course, we also need to impose boundary conditions. To make this precise, we need to define the spatial differential operators.

Definition 2.1. We denote by $\dot{C}_{\infty}(\Omega)$ the space of arbitrarily differentiable functions with compact support in $\Omega$. Then we define the operator grad as the closure of

$$
\begin{aligned}
\stackrel{\circ}{C}_{\infty}(\Omega) \subseteq L^{2}(\Omega) & \rightarrow L^{2}(\Omega)^{3} \\
\phi & \mapsto\left(\partial_{1} \phi, \partial_{2} \phi, \partial_{3} \phi\right)
\end{aligned}
$$

as well as div as the closure of

$$
\begin{aligned}
\stackrel{\circ}{C}_{\infty}(\Omega)^{3} \subseteq L^{2}(\Omega)^{3} & \rightarrow L^{2}(\Omega) \\
\left(\phi_{1}, \phi_{2}, \phi_{3}\right) & \mapsto \sum_{i=1}^{3} \partial_{i} \phi_{i}
\end{aligned}
$$

Integration by parts shows $\operatorname{div} \subseteq-(\operatorname{grad})^{*}$ and we set $\operatorname{div}:=-(\operatorname{grad})^{*}$ and $\operatorname{grad}:=-(\text { div })^{*}$. Similarly, we define the operator curl as the closure of

$$
\begin{aligned}
\dot{C}_{\infty}(\Omega)^{3} \subseteq L^{2}(\Omega)^{3} & \rightarrow L^{2}(\Omega)^{3} \\
\left(\phi_{1}, \phi_{2}, \phi_{3}\right) & \mapsto\left(\begin{array}{ccc}
0 & -\partial_{3} & \partial_{2} \\
\text { Johk Wibey } & \text { \&.Sons }
\end{array}\right)\left(\begin{array}{c}
\phi_{1} \\
\phi_{2} \\
\phi_{3}
\end{array}\right)
\end{aligned}
$$


Mathematical

Methods in the

Applied Sciences

A. N. Mulholland et al.

and curl $:=(\text { curl })^{*} \supseteq$ cơrl. Finally, we define Grad and $\operatorname{Div}^{\circ}$ as the closure of

$$
\begin{aligned}
{\stackrel{\circ}{C}(\Omega)^{3} \subseteq L^{2}(\Omega)^{3}} & \rightarrow \operatorname{sym}\left[L^{2}(\Omega)^{3 \times 3}\right] \\
\left(\phi_{1}, \phi_{2}, \phi_{3}\right) & \mapsto \frac{1}{2}\left(\partial_{j} \phi_{i}+\partial_{i} \phi_{j}\right)_{i, j \in\{1,2,3\}}
\end{aligned}
$$

and of

$$
\begin{aligned}
\operatorname{sym}\left[\stackrel{\circ}{C}_{\infty}(\Omega)^{3 \times 3}\right] \subseteq \operatorname{sym}\left[L^{2}(\Omega)^{3 \times 3}\right] & \rightarrow L^{2}(\Omega)^{3} \\
\left(\phi_{i j}\right)_{i, j \in\{1,2,3\}} & \mapsto\left(\sum_{j=1}^{3} \partial_{j} \phi_{i j}\right)_{i \in\{1,2,3\}},
\end{aligned}
$$

respectively and set $\mathrm{Grad}:=-(\text { Div })^{*}$ as well as Div $:=-(\mathrm{Grad})^{*}$. Elements in the domain of the operators marked by a circle satisfy an abstract homogeneous boundary condition, which, in the case of a smooth boundary $\partial \Omega$, can be written as

$$
u=0 \text { on } \partial \Omega
$$

for $u \in D$ (grad) or $u \in D($ Grad),

$$
u \cdot n=0 \text { on } \partial \Omega
$$

for $u \in D$ (div) or $u \in D$ (Div), where $n$ denotes the exterior unit normal vector field on $\partial \Omega$ and

$$
u \times n=0 \text { on } \partial \Omega,
$$

for $u \in D$ (curl).

We will assume that $v=0, E \times n=0$ and $q \cdot n=0$ on the boundary and hence, the block operator in (2.4) will be replaced by

$$
\left(\begin{array}{cccccc}
0 & - \text { Div } & 0 & 0 & 0 & 0 \\
- \text { Grad } & 0 & 0 & 0 & 0 & 0 \\
0 & 0 & 0 & - \text { curl } & 0 & 0 \\
0 & 0 & \text { curl } & 0 & 0 & 0 \\
0 & 0 & 0 & 0 & 0 & \text { div } \\
0 & 0 & 0 & 0 & \text { grad } & 0
\end{array}\right),
$$

which is now a skew-selfadjoint operator on $H$.

To recall the solution theory (as described in the last chapter of [31]) for our simple situation the needed requirement is that $M_{0}$ is selfadjoint and that

$$
\left.\nu M_{0}+\mathfrak{R e} M_{1} \geq c_{0}>0 \text { for all sufficiently large } \nu \in\right] 0, \infty[\text {. }
$$

Equation (2.5) is for example satisfied if $M_{0}$ is strictly positive definite on its range and $\mathfrak{R e} M_{1}$ strictly positive definite on the null space of $M_{0}$.

Remark. Whenever we are not interested in the actual constant $\left.c_{0} \in\right] 0, \infty[$ we shall write for the strict positive definiteness constraint

$$
\mathfrak{R e} T \geq c_{0}
$$

simply 
A. N. Mulholland et al.

So, the general requirement for the problem class under consideration would be written as

$$
M_{0} \text { selfadjoint, } \nu M_{0}+M_{1} \gg 0
$$

for all sufficiently large $\nu \in] 0, \infty[$.

\section{The System Equations of Thermo-Piezo-Electricity.}

In this section we discuss material relations suggested in [24] and derive the structure of the corresponding operators $M_{0}$ and $M_{1}$. Furthermore, we give sufficient conditions on the parameters involved to warrant the solvability condition (2.6). The material relations described in [24] are initially given in the form (where we write $\mathcal{E}=$ Grad $u$ as usual for the strain tensor)

$$
\begin{aligned}
T & =C \mathcal{E}-e E-\lambda \theta, \\
D & =e^{*} \mathcal{E}+\varepsilon E+p \theta, \\
B & =\mu H, \\
\eta & =\lambda^{*} \mathcal{E}+p^{*} E+\alpha \Theta_{0}^{-1} \theta .
\end{aligned}
$$

Here $C \in L\left(\operatorname{sym}\left[L^{2}(\Omega)^{3 \times 3}\right]\right)$ is the elasticity tensor, $\varepsilon, \mu \in L\left(L^{2}(\Omega)^{3}\right)$ are the permittivity and permeability, respectively, $\alpha:=\varrho_{*} c \in L\left(L^{2}(\Omega)\right)$ is the product of the mass density ${ }^{\dagger} \varrho_{*} \in L^{\infty}(\Omega)$ and the specific heat capacity $c \in L\left(L^{2}(\Omega)\right)$ and $\Theta_{0}:$ $\Omega \rightarrow \mathbb{R}$ is the reference temperature which satisfies $\Theta_{0}, \Theta_{0}^{-1} \in L^{\infty}(\Omega)$. The operators $e \in L\left(L^{2}(\Omega)^{3} ; \operatorname{sym}\left[L^{2}(\Omega)^{3 \times 3}\right]\right), \lambda \in$ $L\left(L^{2}(\Omega)\right.$; sym $\left.\left[L^{2}(\Omega)^{3 \times 3}\right]\right), p \in L\left(L^{2}(\Omega) ; L^{2}(\Omega)^{3}\right)$ are coupling parameters. As a first minor adjustment we make the relative temperature $\Theta_{0}^{-1} \theta$ our new unknown temperature function yielding

$$
\begin{aligned}
T & =C \mathcal{E}-e E-\left(\lambda \Theta_{0}\right) \Theta_{0}^{-1} \theta \\
D & =e^{*} \mathcal{E}+\varepsilon E+\left(p \Theta_{0}\right) \Theta_{0}^{-1} \theta \\
B & =\mu H \\
\Theta_{0} \eta & =\left(\Theta_{0} \lambda^{*}\right) \mathcal{E}+\left(\Theta_{0} p^{*}\right) E+\gamma_{0} \Theta_{0}^{-1} \theta,
\end{aligned}
$$

where we have introduced the abbreviation

$$
\gamma_{0}:=\Theta_{0} \alpha
$$

We assume that heat conduction is governed by the Maxwell-Cattaneo-Vernotte modification

$$
\partial_{0} \kappa_{1} q+\kappa_{0}^{-1} q+\operatorname{grad} \theta=0
$$

for operators $\kappa_{0}, \kappa_{1} \in L\left(L^{2}(\Omega)^{3}\right)$. To adapt the material relations to our framework we solve for $\mathcal{E}$ and obtain

$$
\begin{aligned}
\mathcal{E} & =C^{-1} T+C^{-1} e E+C^{-1}\left(\lambda \Theta_{0}\right) \Theta_{0}^{-1} \theta \\
D & =e^{*} C^{-1} T+\left(\varepsilon+e^{*} C^{-1} e\right) E+\left(p \Theta_{0}+e^{*} C^{-1} \lambda \Theta_{0}\right) \Theta_{0}^{-1} \theta \\
B & =\mu H \\
\Theta_{0} \eta & =\Theta_{0} \lambda^{*} C^{-1} T+\left(\Theta_{0} p^{*}+\Theta_{0} \lambda^{*} C^{-1} e\right) E+\left(\gamma_{0}+\Theta_{0} \lambda^{*} C^{-1} \lambda \Theta_{0}\right) \Theta_{0}^{-1} \theta .
\end{aligned}
$$

${ }^{\dagger}$ Throughout, we identify $L^{\infty}-$ functions with their induced John Wiley \& Sons 
Thus, we arrive at a equation of the form (2.4) with

$$
M_{0}:=\left(\begin{array}{cccccc}
\varrho_{*} & 0 & 0 & 0 & 0 & 0 \\
0 & C^{-1} & C^{-1} e & 0 & C^{-1} \lambda \Theta_{0} & 0 \\
0 & e^{*} C^{-1} & \left(\varepsilon+e^{*} C^{-1} e\right) & 0 & \left(p \Theta_{0}+e^{*} C^{-1} \lambda \Theta_{0}\right) & 0 \\
0 & 0 & 0 & \mu & 0 & 0 \\
0 & \Theta_{0} \lambda^{*} C^{-1} & \left(\Theta_{0} p^{*}+\Theta_{0} \lambda^{*} C^{-1} e\right) & 0 & \left(\gamma_{0}+\Theta_{0} \lambda^{*} C^{-1} \lambda \Theta_{0}\right) & 0 \\
0 & 0 & 0 & 0 & 0 & \kappa_{1}
\end{array}\right)
$$

and

$$
M_{1}:=\left(\begin{array}{cccccc}
0 & 0 & 0 & 0 & 0 & 0 \\
0 & 0 & 0 & 0 & 0 & 0 \\
0 & 0 & \sigma & 0 & 0 & 0 \\
0 & 0 & 0 & 0 & 0 & 0 \\
0 & 0 & 0 & 0 & 0 & 0 \\
0 & 0 & 0 & 0 & 0 & \kappa_{0}^{-1}
\end{array}\right)
$$

We need to verify the solvability condition (2.6) for these operators $M_{0}$ and $M_{1}$.

Theorem 3.1. Assume that $\varrho_{*}, \varepsilon, \mu, C, \gamma_{0}$ are selfadjoint and non-negative. Furthermore, we assume $\varrho_{*}, \mu, C, \gamma_{0} \gg 0$ as well as $\nu\left(\varepsilon-\Theta_{0} p \gamma_{0}^{-1} p^{*} \Theta_{0}\right)+\sigma, \nu \kappa_{1}+\kappa_{0}^{-1} \gg 0$ for sufficiently large $\nu>0$. Then, $M_{0}$ and $M_{1}$ satisfy the condition (2.6) and hence, the corresponding problem of thermo-piezo-electricity is well-posed.

Proof. Obviously, $M_{0}$ is selfadjoint. Moreover, since $\varrho_{*}, \mu, \nu \kappa_{1}+\kappa_{0}^{-1} \gg 0$ for sufficiently large $\nu$, the only thing, which is left to show is, that

$$
\nu\left(\begin{array}{ccc}
C^{-1} & C^{-1} e & C^{-1} \lambda \Theta_{0} \\
e^{*} C^{-1} & \varepsilon+e^{*} C^{-1} e & p \Theta_{0}+e^{*} C^{-1} \lambda \Theta_{0} \\
\Theta_{0} \lambda^{*} C^{-1} & \Theta_{0} p^{*}+\Theta_{0} \lambda^{*} C^{-1} e & \gamma_{0}+\Theta_{0} \lambda^{*} C^{-1} \lambda \Theta_{0}
\end{array}\right)+\left(\begin{array}{ccc}
0 & 0 & 0 \\
0 & \sigma & 0 \\
0 & 0 & 0
\end{array}\right) \gg 0
$$

for sufficiently large $\nu$. By symmetric Gauss steps as a congruence transformations we get that the above operator is congruent to

$$
\nu\left(\begin{array}{ccc}
C^{-1} & 0 & 0 \\
0 & \varepsilon & p \Theta_{0} \\
0 & \Theta_{0} p^{*} & \gamma_{0}
\end{array}\right)+\left(\begin{array}{ccc}
0 & 0 & 0 \\
0 & \sigma & 0 \\
0 & 0 & 0
\end{array}\right)
$$

which itself is congruent by another symmetric Gauss step to

$$
\nu\left(\begin{array}{ccc}
C^{-1} & 0 & 0 \\
0 & \varepsilon-\Theta_{0} p \gamma_{0}^{-1} p^{*} \Theta_{0} & 0 \\
0 & 0 & \gamma_{0}
\end{array}\right)+\left(\begin{array}{ccc}
0 & 0 & 0 \\
0 & \sigma & 0 \\
0 & 0 & 0
\end{array}\right)
$$

The latter operator is then strictly positive definite by assumption and so the assertion follows.

Remark.

1. Note that due to the generality of the assumptions then limit cases such as $\varepsilon=\Theta_{0} p \gamma_{0}^{-1} p^{*} \Theta_{0}$ and $\sigma \gg 0$ (eddy current case) are covered by the theorem. 
2. To provide a hint towards further generalizations of the specific model we incorporate for example piezo-magnetic effects by adding in a corresponding coupling term. That is, we replace $M_{0}$ by the operator

$$
\left(\begin{array}{cccccc}
\varrho_{*}+\beta \mu \beta^{*} & 0 & 0 & -\beta \mu & 0 & 0 \\
0 & C^{-1} & C^{-1} e & 0 & C^{-1} \lambda \Theta_{0} & 0 \\
0 & e^{*} C^{-1} & \left(\varepsilon+e^{*} C^{-1} e\right) & 0 & \left(p \Theta_{0}+e^{*} C^{-1} \lambda \Theta_{0}\right) & 0 \\
-\mu \beta^{*} & 0 & 0 & \mu & 0 & 0 \\
0 & \Theta_{0} \lambda^{*} C^{-1} & \left(\Theta_{0} p^{*}+\Theta_{0} \lambda^{*} C^{-1} e\right) & 0 & \left(\gamma_{0}+\Theta_{0} \lambda^{*} C^{-1} \lambda \Theta_{0}\right) & 0 \\
0 & 0 & 0 & 0 & 0 & \kappa_{1}
\end{array}\right),
$$

where $\beta \in L\left(L^{2}(\Omega)^{3}\right)$ is a further parameter, which now couples the displacement field u with the magnetic field $H$. Note that to match up with the piezo-magnetic model discussed in [12] we have to introduce a new composite magnetic field

$$
\widetilde{H}:=\beta^{*} v+H
$$

in place of $H$ as one of the basic unknowns. The well-posedness conditions remain unchanged.

\section{A “Simplification”.}

The above situation is commonly "simplified" by replacing the full Maxwell equations by the static Maxwell equations for the electric field; the so called quasi-electrostatic approach. There is a price to be paid for this modification, which made us use quotation marks around the term "simplify " and "simplification". We assume that $E=-\operatorname{grad} \varphi$ for a suitable potential $\varphi \in D(\operatorname{grad})$ and $D \in D(\operatorname{div})$ and we set $\psi:=\operatorname{div} D$. Moreover, we assume that there is no conductivity term, i.e. $\sigma=0$ and thus, the system under consideration reduces to

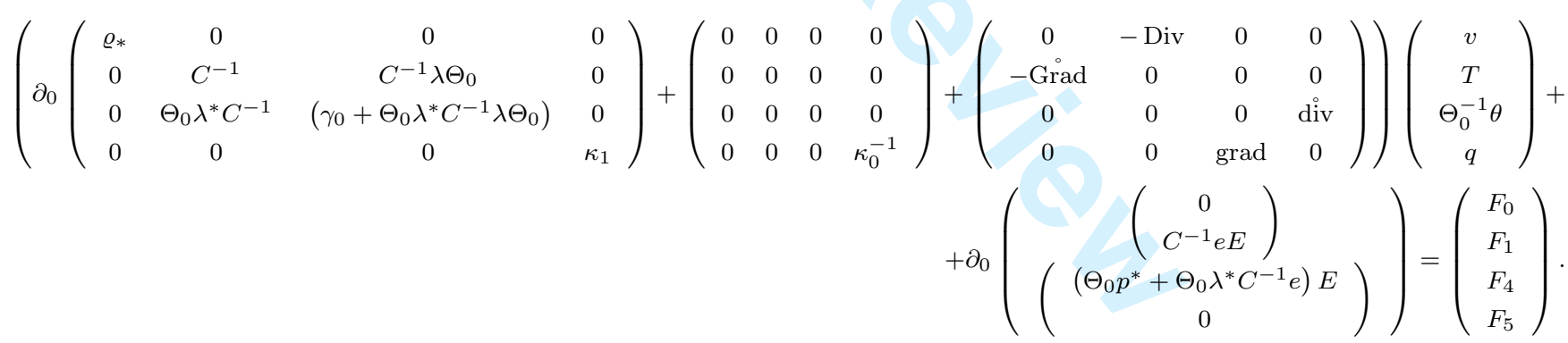

We have now to express $E$ in terms of the other unknowns as part of a new material law. Recall, that we have

$$
D=e^{*} C^{-1} T+\left(\varepsilon+e^{*} C^{-1} e\right) E+\left(p \Theta_{0}+e^{*} C^{-1} \lambda \Theta_{0}\right) \Theta_{0}^{-1} \theta
$$

By setting

$$
\begin{aligned}
\Phi & :=e^{*} C^{-1} T+\left(p \Theta_{0}+e^{*} C^{-1} \lambda \Theta_{0}\right)\left(\Theta_{0}^{-1} \theta\right) \\
& =e^{*} C^{-1}(T+\lambda \theta)+p \theta,
\end{aligned}
$$

$D$ can be written as

$$
D=\left(\varepsilon+e^{*} C^{-1} e\right) E+\Phi .
$$

Using now that $\psi=\operatorname{div} D$ and $E=-\operatorname{grad} \varphi$ we get that 
Mathematical

Methods in the

Applied Sciences

A. N. Mulholland et al.

We assume that $C, \varepsilon$ are selfadjoint and $\varepsilon+e^{*} C^{-1} e \gg 0$ and set $M:=\sqrt{\varepsilon+e^{*} C^{-1} e}$. Then, the latter equality can be written as

$$
-\operatorname{div} M^{2} \operatorname{grad} \varphi+\operatorname{div} M M^{-1} \Phi=\psi,
$$

which gives

$$
\operatorname{grad} \varphi+M^{-1}\left((M \operatorname{grad})|M \operatorname{grad}|^{-2} \operatorname{div} M\right) M^{-1} \Phi=M^{-1}(M \operatorname{grad}) \mid M \text { grad }\left.\right|^{-2} \psi,
$$

if we assume that ${ }^{\ddagger} \psi \in D\left(\mid M\right.$ grad $\left.\left.\right|^{-2}\right)$. This suggests to replace

$$
\begin{aligned}
E & =-\operatorname{grad} \varphi \\
& =M^{-1}\left(\overline{(M \text { grad }) \mid M \text { grad }\left.\right|^{-2} \operatorname{div} M}\right) M^{-1} \Phi-M^{-1}(M \text { grad }) \mid M \text { grad }\left.\right|^{-2} \psi,
\end{aligned}
$$

where we use the closure bar to ensure this operator is in $L\left(L^{2}(\Omega)^{3}\right)$. Indeed, this operator is not only bounded but also an orthogonal projector as the next lemma shows.

Lemma] 4.1. Let $A: D(A) \subseteq H_{0} \rightarrow H_{1}$ be a densely defined and closed linear operator between two Hilbert spaces $H_{0}, H_{1}$ such that $A^{*} A$ is injective. Then

$$
\overline{A\left(A^{*} A\right)^{-1} A^{*}}=P_{\overline{A\left[H_{0}\right]}},
$$

the orthogonal projector on the closure of the range of $A$.

Proof. Let $x \in D\left(A^{*}\right)$ and set

$$
f:=A\left(A^{*} A\right)^{-1} A^{*} x .
$$

Then, obviously, $f \in A\left[H_{0}\right]$ and $f \in D\left(A^{*}\right)$. Since $\overline{A\left[H_{0}\right]}=\left([\{0\}] A^{*}\right)^{\perp}$, we get that

$$
A^{*} P_{\overline{A\left[H_{0}\right]}} x=A^{*} A\left(A^{*} A\right)^{-1} A^{*} x=A^{*} f
$$

and so

$$
A^{*}\left(P_{\overline{A\left[H_{0}\right]}} x-f\right)=0
$$

which implies

$$
P_{\overline{A\left[H_{0}\right]}} x-f \in[\{0\}] A^{*} \cap \overline{A\left[H_{0}\right]}=\{0\} .
$$

Thus,

$$
P_{\overline{A\left[H_{0}\right]}} x=A\left(A^{*} A\right)^{-1} A^{*} x
$$

for each $x \in D\left(A^{*}\right)$ and thus, the assertion follows by the density of $D\left(A^{*}\right)$.

$$
\begin{aligned}
& \text { Using this result for } A:=M \text { grad and setting } P:=P_{\overline{A\left[L^{2}(\Omega)\right]}} \text { we get that } \\
& \qquad E=-M^{-1} P M^{-1} \Phi-M^{-1}(M \text { grad }) \mid M \text { grad }\left.\right|^{-2} \psi .
\end{aligned}
$$

\footnotetext{
${ }^{\ddagger}$ Note that if $\Omega$ is bounded, this condition is always satisfied since in this \& Sons grad is onto.
} 
Hence, the last term on the left hand side in (4.1) can be replaced by

$$
\begin{aligned}
& \left(\begin{array}{c}
\left(\begin{array}{c}
0 \\
C^{-1} e E
\end{array}\right) \\
\left(\begin{array}{c}
\left(\Theta_{0} p^{*}+\Theta_{0} \lambda^{*} C^{-1} e\right) E \\
0
\end{array}\right)
\end{array}\right)-\left(\begin{array}{c}
0 \\
\left(\begin{array}{c}
C^{-1} e M^{-1} P M^{-1} \Phi
\end{array}\right) \\
=-\left(\begin{array}{c}
0 \\
\left(\Theta_{0}^{-1} e p^{-1}(M \operatorname{orad}) \mid M \text { grad }\left.\right|^{-2} \psi\right.
\end{array}\right) \\
\left(\Theta_{0} \lambda^{*} C^{-1} e\right) M^{-1} P M^{-1} \Phi \\
0
\end{array}\right) .
\end{aligned}
$$

Using now the definition of $\Phi$, we can write

$$
\begin{aligned}
& -\left(\begin{array}{c}
\left(\begin{array}{c}
0 \\
C^{-1} e M^{-1} P M^{-1} \Phi
\end{array}\right) \\
0
\end{array}\right)
\end{aligned}
$$

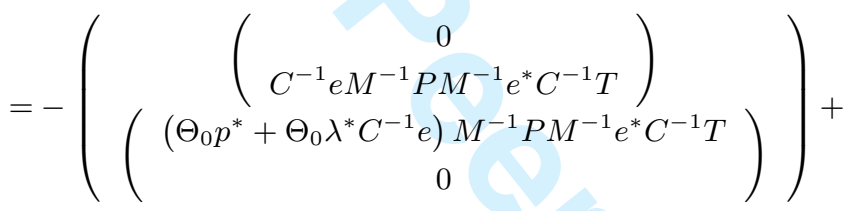

$$
\begin{aligned}
& -\left(\begin{array}{c}
\left(\begin{array}{c}
0 \\
C^{-1} e M^{-1} P M^{-1}\left(p \Theta_{0}+e^{*} C^{-1} \lambda \Theta_{0}\right)\left(\Theta_{0}^{-1} \theta\right)
\end{array}\right) \\
0
\end{array}\left(\begin{array}{c}
\left(\Theta_{0} p^{*}+\Theta_{0} \lambda^{*} C^{-1} e\right) M^{-1} P M^{-1}\left(p \Theta_{0}+e^{*} C^{-1} \lambda \Theta_{0}\right)\left(\Theta_{0}^{-1} \theta\right) \\
0
\end{array}\right)\right. \\
& =-W_{0}\left(\begin{array}{c}
v \\
T \\
\Theta_{0}^{-1} \theta \\
q
\end{array}\right)
\end{aligned}
$$

with $W_{0}$ given by

$$
W_{0}:=\left(\begin{array}{cccc}
0 & 0 & 0 & 0 \\
0 & C^{-1} e M^{-1} P M^{-1} e^{*} C^{-1} & C^{-1} e M^{-1} P M^{-1}\left(p \Theta_{0}+e^{*} C^{-1} \lambda \Theta_{0}\right) & 0 \\
0 & \left(\Theta_{0} p^{*}+\Theta_{0} \lambda^{*} C^{-1} e\right) M^{-1} P M^{-1} e^{*} C^{-1} & \left(\Theta_{0} p^{*}+\Theta_{0} \lambda^{*} C^{-1} e\right) M^{-1} P M^{-1}\left(p \Theta_{0}+e^{*} C^{-1} \lambda \Theta_{0}\right) & 0 \\
0 & 0 & 0 & 0
\end{array}\right)
$$

Summarizing, Equation (4.1) reads as

$\partial_{0}\left(\left(\begin{array}{cccc}\varrho_{*} & 0 & 0 & 0 \\ 0 & M_{11} & M_{12} & 0 \\ 0 & M_{12}^{*} & M_{22} & 0 \\ 0 & 0 & 0 & \kappa_{1}\end{array}\right)+\left(\begin{array}{cccc}0 & 0 & 0 & 0 \\ 0 & 0 & 0 & 0 \\ 0 & 0 & 0 & 0 \\ 0 & 0 & 0 & \kappa_{0}^{-1}\end{array}\right)+\left(\begin{array}{cccc}0 & -\operatorname{Div} & 0 & 0 \\ -\operatorname{Grad} & 0 & 0 & 0 \\ 0 & 0 & 0 & \operatorname{div} \\ 0 & 0 & \operatorname{grad} & 0\end{array}\right)\right)\left(\begin{array}{c}v \\ T \\ \Theta_{0}^{-1} \theta \\ q\end{array}\right)=G$, 
with

$$
\begin{aligned}
M_{11} & :=C^{-1}-C^{-1} e M^{-1} P M^{-1} e^{*} C^{-1} \\
M_{12} & :=C^{-1} \lambda \Theta_{0}-C^{-1} e M^{-1} P M^{-1}\left(p \Theta_{0}+e^{*} C^{-1} \lambda \Theta_{0}\right) \\
& =M_{11} \lambda \Theta_{0}-C^{-1} e M^{-1} P M^{-1} p \Theta_{0} \\
M_{22} & :=\left(\gamma_{0}+\Theta_{0} \lambda^{*} C^{-1} \lambda \Theta_{0}\right)-\left(\Theta_{0} p^{*}+\Theta_{0} \lambda^{*} C^{-1} e\right) M^{-1} P M^{-1}\left(p \Theta_{0}+e^{*} C^{-1} \lambda \Theta_{0}\right)
\end{aligned}
$$

and the right-hand side has to be adjusted to

$$
G:=\left(\begin{array}{c}
F_{0} \\
\left.F_{1}+C^{-1} e M^{-1}(M \operatorname{\circ g}) \mid M \operatorname{grad}\right) \\
F_{4}+\left(\Theta_{0} p^{*}+\Theta_{0} \lambda^{*} C^{-1} e\right) M^{-1}(M \operatorname{grad})|M \operatorname{grad}|^{-2} \partial_{0} \psi \\
F_{5}
\end{array}\right)
$$

where we additionally assume that $\partial_{0} \psi \in D\left(\mid M\right.$ grad $\left.\left.\right|^{-2}\right)$. In the next theorem we provide sufficient conditions on the operators involved in order to obtain a well-posedness result for (4.3).

Theorem 4.2. Let $C, M, \varrho_{*}, \kappa_{1}$ be selfadjoint and non-negative such that $C, M, \varrho_{*}, \nu \kappa_{1}+\kappa_{0}^{-1} \gg 0$ for sufficiently large $\nu$ and $P$ be an orthogonal projector. We set $Q:=P M^{-1} e^{*} C^{-\frac{1}{2}}$ and assume that

$$
\begin{array}{r}
1-Q^{*} Q \gg 0, \\
\gamma_{0}-\Theta_{0} p^{*} M^{-1} P\left(1-Q Q^{*}\right)^{-1} P M^{-1} p \Theta_{0} \gg 0 .
\end{array}
$$

Then, Equation (4.3) with $M_{11}, M_{12}, M_{22}$ given by (4.4) is well-posed.

Proof. We need to verify the solvability condition (2.6). To do so, it suffices to consider the block operator sub-matrix

$$
\left(\begin{array}{cc}
M_{11} & M_{12} \\
M_{12}^{*} & M_{22}
\end{array}\right)
$$

Noting that $M_{11}=C^{-1}-C^{-\frac{1}{2}} Q^{*} Q C^{-\frac{1}{2}}=C^{-\frac{1}{2}}\left(1-Q^{*} Q\right) C^{-\frac{1}{2}}$, we obtain that $M_{11}$ is boundedly invertible. Hence, by applying a symmetric Gauss step, we are led to consider the matrix

$$
\left(\begin{array}{cc}
M_{11} & 0 \\
0 & M_{22}-M_{12}^{*} M_{11}^{-1} M_{12}
\end{array}\right)
$$

which is strictly positive definite if and only if $M_{22}-M_{12}^{*} M_{11}^{-1} M_{12} \gg 0$. We have

$$
\begin{aligned}
M_{22}= & \left(\gamma_{0}+\Theta_{0} \lambda^{*} C^{-1} \lambda \Theta_{0}\right)-\left(\Theta_{0} p^{*}+\Theta_{0} \lambda^{*} C^{-1} e\right) M^{-1} P M^{-1}\left(p \Theta_{0}+e^{*} C^{-1} \lambda \Theta_{0}\right) \\
= & \left(\gamma_{0}+\Theta_{0} \lambda^{*} C^{-1} \lambda \Theta_{0}\right)- \\
& -\left(\Theta_{0} p^{*} M^{-1} P M^{-1} p \Theta_{0}+2 \mathfrak{R e}\left(\Theta_{0} p^{*} M^{-1} Q C^{-\frac{1}{2}} \lambda \Theta_{0}\right)+\Theta_{0} \lambda^{*} C^{-\frac{1}{2}} Q^{*} Q C^{-\frac{1}{2}} \lambda \Theta_{0}\right) \\
= & \gamma_{0}+\Theta_{0} \lambda^{*}\left(C^{-1}-C^{-\frac{1}{2}} Q^{*} Q C^{-\frac{1}{2}}\right) \lambda \Theta_{0}- \\
& -\left(\Theta_{0} p^{*} M^{-1} P M^{-1} p \Theta_{0}+2 \mathfrak{R e}\left(\Theta_{0} p^{*} M^{-1} Q C^{-\frac{1}{2}} \lambda \Theta_{0}\right)\right) \\
= & \gamma_{0}+\Theta_{0} \lambda^{*} M_{11} \lambda \Theta_{0}-\left(\Theta_{0} p^{*} M^{-1} P M^{-1} p \Theta_{0}+2 \mathfrak{R e}\left(\Theta_{0} p^{*} M^{-1} Q C^{-\frac{1}{2}} \lambda \Theta_{0}\right)\right)
\end{aligned}
$$

and

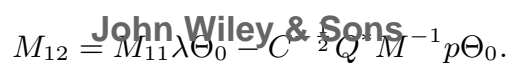


Thus,

$$
\begin{aligned}
M_{12}^{*} M_{11}^{-1} M_{12} & =M_{12}^{*}\left(\lambda \Theta_{0}-M_{11}^{-1} C^{-\frac{1}{2}} Q^{*} M^{-1} p \Theta_{0}\right) \\
& =\Theta_{0} \lambda^{*} M_{11} \lambda \Theta_{0}-2 \Re \mathfrak{R e}\left(\Theta_{0} \lambda^{*} C^{-\frac{1}{2}} Q^{*} M^{-1} p \Theta_{0}\right)+\Theta_{0} p^{*} M^{-1} Q C^{-\frac{1}{2}} M_{11}^{-1} C^{-\frac{1}{2}} Q^{*} M^{-1} p \Theta_{0}
\end{aligned}
$$

and hence, we get

$$
M_{22}-M_{12}^{*} M_{11}^{-1} M_{12}=\gamma_{0}-\Theta_{0} p^{*}\left(M^{-1} P M^{-1}+M^{-1} Q C^{-\frac{1}{2}} M_{11}^{-1} C^{-\frac{1}{2}} Q^{*} M^{-1}\right) p \Theta_{0}
$$

Using $M_{11}^{-1}=C^{\frac{1}{2}}\left(1-Q^{*} Q\right)^{-1} C^{\frac{1}{2}}$ we obtain

$$
Q C^{-\frac{1}{2}} M_{11}^{-1} C^{-\frac{1}{2}} Q^{*}=Q\left(1-Q^{*} Q\right)^{-1} Q^{*}=-1+\left(1-Q Q^{*}\right)^{-1}
$$

and since $Q=P Q$ we have

$$
Q C^{-\frac{1}{2}} M_{11}^{-1} C^{-\frac{1}{2}} Q^{*}=-P+P\left(1-Q Q^{*}\right)^{-1} P
$$

and thus,

$$
\begin{aligned}
M_{22}-M_{12}^{*} M_{11}^{-1} M_{12} & =\gamma_{0}-\Theta_{0} p^{*}\left(M^{-1} P M^{-1}+M^{-1}\left(-P+P\left(1-Q Q^{*}\right)^{-1} P\right) M^{-1}\right) p \Theta_{0} \\
& =\gamma_{0}-\Theta_{0} p^{*} M^{-1} P\left(1-Q Q^{*}\right)^{-1} P M^{-1} p \Theta_{0}
\end{aligned}
$$

which is strictly positive definite by assumption.

If we apply the latter theorem with $M=\sqrt{\varepsilon+e^{*} C^{-1} e}, P=P \overline{M \operatorname{grad}\left[L^{2}(\Omega)\right]}$ and $G$ as given in (4.5), where we assume that $\partial_{0} \psi \in D\left(|M \operatorname{grad}|^{-2}\right)$, we obtain a well-posedness result for the system described in $(4.1), \operatorname{where} E=-\operatorname{grad} \varphi, D=\operatorname{div} \psi$ and $D$ and $E$ are coupled by (4.2).

\section{Conclusion.}

The mathematical modelling of piezoelectric transducers can de-risk the development of new sensors and actuators and, coupled with the widespread availability of powerful computing facilities, there is an increasing reliance on this approach. These models are typically used in inverse problems associated with obtaining a set of optimal design parameters for a desired set of sensor operating characteristics. It is therefore vital that these models are examined for their well-posedness. There has been a steady body of work considering this problem over recent years and this paper has extended this to consider the case when the thermal effects are coupled to the piezoelectric equations. This is motivated by the need to develop sensors that can operate at high temperatures in, for example, the non-destructive testing of heat exchanger surfaces in nuclear energy plants. A modelling assumption that is often employed in order to reduce the size of the model is the so-called quasi-electrostatic approach. Given the widespread use of this approximation the well-posedness of the quasi-electrostatic model of a piezoelectric material was also shown in this paper.

\section{References}

1. Akamatsu, M. \& Nakamura, G., Well-Posedness of Initial-Boundary Value Problems for Piezoelectric Equations, Applicable Analysis, 81, 129-141, (2002).

2. Algehyne, E.A. \& Mulholland, A.J., A finite element approach to modelling fractal ultrasonic transducers, IMA J Appl Math, first published online May 29, 2015, doi:10.1093/imamat/hxv012, (2015).

3. Ammari, K. \& Nicaise, S., Stabilization of a piezoelectric system, arXiv preprint arXiv:1005.2545, (2010). 
Mathematical

Methods in the

Applied Sciences

A. N. Mulholland et al.

4. Ionica, A., Nicusor, C. \& Andaluzia, M., Antiplane shear deformation of piezoelectric bodies in contact with a conductive support, J Glob Optim, 56, 103-119, (2013) .

5. Barboteu, M. \& Sofonea, M., Solvability of a dynamic contact problem between a piezoelectric body and a conductive foundation, Applied Mathematics and Computation, 215, 2978-2991, (2009).

6. Benaissa, H., Essoufi,EL-H. \& Fakhar, R., Existence results for unilateral contact problem with friction of thermo-electro-elasticity, Appl. Math. Mech.-Engl. Ed., 36(7), 911-926, (2015).

7. Bonaldi, F., Geymonat, G., \& Krasucki, F., Modelling of smart materials with thermal effects: dynamic and quasi-static evolution, hal-01167897, (2015).

8. P. G. Ciarlet. Mathematical Elasticity, volume II: Theory of Plates, volume 27 of Studies in mathematics and its applications. Elsevier Science B. V., Amsterdam, 1997.

9. Chkadua, G. \& Natroshvili, D., Interaction of acoustic waves and piezoelectric structures, Mathematical Methods in the Applied Sciences, 38(11), 2149-2170, (2015).

10. Daros, C.H. \& Antes, H., The elastic motion of a transversely isotropic, piezoelectric solid caused by impulsive loading, $Z$. angew. Math. Phys., 51, 397-418, (2000).

11. Fang, D. \& Liu, J., Fracture Mechanics of Piezoelectric and Ferroelectric Solids, Springer-Verlag Berlin, (2013).

12. Ferreira, M. V. and Perla Menzala, G., Uniform stabilization of an electromagnetic-elasticity problem in exterior domains. Discrete Contin. Dyn. Syst., 18(4):719-746, 2007.

13. Imperiale, S. \& Joly, P., Mathematical and Numerical Modelling of Piezoeletric Sensors, ESAIM: M2AN 46, 875-909, (2012).

14. Jiang, L.Z., Integral representation and Green's functions for 3D time-dependent thermo-piezoelectricity, Int. J. Solids and Structures, 37, 6155-6171, (2000).

15. Kaltenbacher, B., Lahmer, T., Mohr, M.. \& Kaltenbacher, M., PDE based determination of piezoelectric material tensors, Euro. J. of Applied Mathematics, 17, 383-416, (2006).

16. Kapitonov, B. \& Perla Menzala, G., Uniform Stabilization and Exact Control of a Mulitlayered Piezoelectric Body, Portugaliae Mathematica, 60(4), 411-454, (2003).

17. Kapitonov, B., Miara, B. \& Perla Menzala, G., Stabilization of a Layered Piezoelectric 3-D Body by Boundary Dissipation, ESAIM: COCV, 12, 198-215, (2006).

18. Kapitonov, B., Miara, B. \& Perla Menzala, G., Boundary Observation and Exact Control of a Quasi-Electrostatic Piezoelectric System in Multilayered Media, SIAM J. Control Optim. 46(3), 1080-1097, (2007).

19. Lahmer, T., Kaltenbacher, B. \& Schulz, V., Optimal measurement selection for piezoelectric material tensor identification, Inverse Problems in Science and Engineering, 16(3), 369-387, (2008).

20. Leugering, G. \& Nazarov, S.A., The Eshelby Theorem and its Variants for Piezoelectric Media, Arch. Rational Mech. Anal. 215, 707-739, (2015).

21. Mercier, D. \& Nicaise, S., Existence, Uniqueness, and Regularity Results for Piezoelectric Systems, SIAM J. Math. Anal., 37(2), 651-672, (2005).

22. Miara, B. \& Santos, M.L., Energy decay in piezoelectric systems, Applicable Analysis, 88(7), 947-960, (2009).

23. Migorski, S., Ochal, A. \& Sofonea, M., Weak solvability of a piezoelectric contact problem, Euro. J. Applied Mathematics, 20, 145-167, (2009).

24. Mindlin, R.D., Equations Of High Frequency Vibrations of Thermo-Piezoelectric Crystal Plates, Intl. J. Solids 8 Structures, 10, 625-637, (1974).

25. Mulholland A.J., Ramadas S.N., O'Leary R.L., Parr A., Troge A., Pethrick R.A. \& Hayward G., A Theoretical Analysis of a Piezoelectric Ultrasound Device with an Active Matching Layer, Ultrasonics, 47(1), 102-110, (2007).

26. Mulholland A.J., Ramadas S.N., O’Leary R.L., Parr A., A.Troge A., Pethrick R.A. \& Hayward G., Enhancing the performance of piezoelectric ultrasound transducers by the use of multiple matching layers, IMA J. Appl. Maths., 73, 936-949, (2008).

27. Orr L-A., Mulholland A.J., O'Leary R.L. \& Hayward G., Harmonic Analysis of Lossy Piezoelectric Composite Transducers using the Plane Wave Expansion Method, Ultrasonics, 48(8), 652-663, (2008).

28. Orr L-A., Mulholland A.J., O'Leary R.L., Parr A, Pethrick R.A. \& Hayward G., Theoretical Modelling of Frequency Dependent Elastic Loss in Composite Ultrasonic Transducers, Ultrasonics, 47(1), 130-137, (2007).

29. Orr L-A., Mulholland A.J., O’Leary R.L., Pethrick R.A. \& Hayward G., Analysis of Ultrasonic Transducers with Fractal Architecture, Fractals, 16(4), 222-349, (2008).

30. Perla Menzala, G. \& Sejje Surez, J., Uniform stabilization of a thermopiezoelectric/piezomagnetic model, J.. Math. Anal. Appl., 409, 56-73, (2014).

31. R. Picard and D. F. McGhee. Partial Differential Equations: A unified Hilbert Space Approach, volume 55 of De Gruyter Expositions in Mathematics. De Gruyter. Berlin, New York. 518 p., 2011.

32. Rafiee, M., Mohammadi, M., Sobhani Aragh, B.. \&ohaph Wipiey ${ }_{\&}$ Sonlinear free and forced thermo-electro-aero-elastic vibration 


\section{A. N. Mulholland et al.}

and dynamic response of piezoelectric functionally graded laminated composite shells, Part I: Theory and analytical solutions, Composite Structures, 103, 179-187, (2013).

33. M. Rahmoune, A. Benjeddou, R. Ohayon, and D. Osmont. New thin piezoelectric plate models. Journal of Intelligent Material Systems and Structures, 9(12):1017-1029, 1998.

34. Sixto-Camacho, J.M., Bravo-Castillero, J., Brenner, R., Guinovart-Daz, R., Mechkour, H., Rodrguez-Ramos, R., \& Sabina, F.J., Asymptotic homogenization of periodic thermo-magneto-electro-elastic heterogeneous media, Computers and Mathematics with Applications, 66, 2056-2074, (2013).

35. Tant,K.M.M., Mulholland, A.J., \& Gachagan, A., A Model-Based Approach to Crack Sizing With Ultrasonic Arrays, IEEE TUFFC, 62(5), 915-926, (2015).

36. Tant, K.M.M., Mulholland, A.J., Langer, M., \& Gachagan, A., A fractional Fourier transform analysis of the scattering of ultrasonic waves, Proc. R. Soc. A, 471 (2176), 20140958, DOI: 10.1098/rspa.2014.0958, (2015).

37. Tramontana, M., Gachagan, A., Nordon, A.., Littlejohn, D. \& Mulholland, A.J., System Modeling and Device Development for Passive Acoustic Monitoring of a Particulate-Liquid Process, Sensors 83 Actuators: A. Physical, 228, 159-169, (2015) .

38. Tucsnak, M., Regularity and Exact Controllability for a Beam with Piezoelectric Actuator, SIAM J. Control and Optimization, 34(3), 922-930, (1996).

39. Walker, A.J. \& Mulholland, A.J., A theoretical model of an ultrasonic transducer incorporating spherical resonators, IMA J Appl Math, first published online August 18, 2015 doi:10.1093/imamat/hxv023, (2015).

40. Walker A.J. \& Mulholland A.J., Piezoelectric Ultrasonic Transducers with Fractal Geometry, Fractals, 19(4), 469-479, (2011).

41. Walker A.J., Mulholland A.J. \& Whitely S., A Theoretical Model of an Electrostatic Ultrasonic Transducer incorporating Resonating Conduits, IMA J. Appl. Maths, 75(5), 796-810, (2010).

42. Wynn, L.T., Truitt, A., Heim, I. \& Nima Mahmoodi, S., Modeling and Response Analysis of Piezoelectric Flag In Wind Flow, Proc. ASME 2013 Dynamic Systems and Control Conference DSCC2013, October 21-23, 2013, Palo Alto, California, USA, DSCC20133912, (2013).

43. Yuan, X. and Yang, F., Energy Transfer in Pyroelectric Material, Heat Conduction - Basic Research, Prof. Vyacheslav Vikhrenko (Ed.), ISBN: 978-953-307-404-7, InTech, DOI: 10.5772/26053, (2011). 